\title{
Arbeiten zum Datenschutz im IT-Grundschutz vorläufig abgeschlossen
}

Claus Simon

\begin{abstract}
Nach mehreren Ansätzen in den vergangenen Jahren ist es jetzt gelungen, die Arbeiten zur Integration des Datenschutzes in den IT-Grundschutz zu einem Abschluss zu bringen. Nach den Datenschutzbeauftragten des Bundes und der Länder haben jetzt auch die Aufsichtsbehörden für den nichtöffentlichen Bereich untereinander die Arbeitsentwürfe abgestimmt. Die gemeinsam erarbeiteten Endfassungen liegen jetzt vor.
\end{abstract}

\section{Baustein 1.5}

Wichtigstes Ergebnis ist ein Baustein 1.5 „Datenschutz“ als Ergänzung für die ITGrundschutzkataloge des Bundesamtes für die Informationssicherheit (BSI). Dieser Baustein enthält eine einleitende Beschreibung des Datenschutzes, seiner verschiedenen Facetten und der für unterschiedliche Bereiche zuständigen Kontrollinstanzen, was zur Sensibilisierung der Managementprozesse und ihrer zugehörigen Teams dienen soll. Wie durch die Struktur der anderen Bausteine der IT-Grundschutzkataloge vorgegeben, folgen dann besondere datenschutzrechtliche Gefährdungen und die zugehörigen Maßnahmen, um diesen Gefährdungen möglichst begegnen $\mathrm{zu}$ können. Eine besondere Maßnahme stellt dabei die Beschreibung eines eigenständigen Datenschutz-Management-Prozesses dar. Diese Beschreibungen sind ergänzt durch geeignete Tabellen, mit denen die Relationen zwischen den durch Datenschutzgesetze vorgegebenen, allgemeinen Kontrollzielen und den konkreten Maßnahmen des IT-Grundschutzes einschließlich der neuen Datenschutz-Maßnahmen aufgezeigt werden.

Der Baustein 1.5 „Datenschutz“ soll nach entsprechender redaktioneller Überar- beitung bis Mitte des Jahres auf der Website des BSI für zum Abruf bereitgestellt werden. Eine Integration des Bausteins in die IT-Grundschutzkataloge direkt ist nicht sinnvoll, da diese für eine internationale Zertifizierung ausgelegt sind. Allerdings wird in die IT-Grundschutzkataloge ein kurzer Verweistext auf den Baustein und seine Fundstelle aufgenommen. Da sich der Baustein an der deutschen Gesetzgebung zum Datenschutz ausrichtet, wird er zwar nicht übersetzt, kann aber sinngemäß auch im Ausland angewandt werden. Der Baustein kann als Vorab-Version jetzt schon beim BfDI (www.bfdi.bund.de) oder beim LfDI-Saarland (www.lfdi.saarland.de) abgerufen werden.

Als weiteres Ergebnis wurden Vorschläge zur datenschutzrechtlichen Ergänzung der bisher schon bestehenden IT-Grundschutzkataloge erarbeitet. Hierzu gehört auch eine aktuelle Definition der Begriffe „Datenschutz“ und „Datenschutzmanagement". Eine Veröffentlichung dieser Neufassung der IT-Grundschutz-Kataloge einschließlich der Bereitstellung in der Website durch das BSI ist für Oktober 2007 vorgesehen.

\section{Datenschutzmanagement}

In Abstimmung mit dem BSI wurden außerdem Datenschutz-Ergänzungen für die BSI-Standards erarbeitet, die den ITGrundschutzkatalogen übergeordnet sind. Im BSI-Standard 100-I „Managementsysteme für Informationssicherheit" kommen Aspekte wie „Datenschutz als Teil der Management-Prinzipien“, „Beteiligung des Datenschutzbeauftragten“" und „Mitarbeiterschulung zum Datenschutz" hinzu.

Im BSI-Standard 100-II „IT-Grundschutz-Vorgehensweise“ wurde im Schutz- stufenkonzept der „Schutzbedarf unter Berücksichtigung des Datenschutzes“ präzisiert sowie der „Datenschutzbeauftragte als Organisationseinheit“ und seine „Anbindung als Stabstelle bei der Behörden- bzw. Unternehmensleitung“ herausgestellt. Neben einer „Aufgabenbeschreibung des Datenschutzbeauftragten“" wurden auch die „Datenschutzkontrolle als begleitende Aufgabe der Revision“ und eine ,,datenschutzgerechte Dokumentation“ ergänzt. Das BSI beabsichtigt, Ende des Jahres entsprechend überarbeitete Fassungen dieser beiden Standards vorzulegen.

\section{Ausblick}

Für die Zukunft ist angedacht, den Datenschutzbaustein in Kooperation mit dem BSI und seinen IT-Grundschutz-Auditoren fortzuentwickeln. Zusätzlich könnten Fassungen erarbeiten werden, die sowohl bei nationaler als auch internationaler Auditierung/Zertifizierung zugrunde gelegt werden können

Claus Simon

Referatsleiter Technik

beim Landesbeauftragten für Datenschutz

und Informationsfreiheit

Fritz-Dobisch-Str. 1266111 Saarbrücken

Postfach $102631 \quad 66026$ Saarbrücken

Tel.: 06819478136

Fax: 06819478129

simon@lfdi.saarland.de

\author{
Links zum Download \\ - www.datenschutz.de/technik/ \\ - http://www.lfdi.saarland.de/html \\ /lfd-internet/info-grundschutz.htm \\ $\checkmark$ www.bfdi.bund.de
}

